\title{
Ex-Vivo Ureteroscopy at the Time of Live Donor Nephrectomy
}

\author{
George R. Schade, M.D., J. Stuart Wolf, Jr., M.D., and Gary J. Faerber, M.D.
}

\begin{abstract}
Background and Purpose: Potential transplant renal allograft recipients exceed the number of donors. Our institution now considers patients with small, unilateral, nonobstructing, incidental renal calculi for possible renal donation. We adopted ex-vivo ureteroscopy (ExURS) to render these kidneys stone free at the time of renal transplantation. We examined the safety and efficacy of ExURS.

Patients and Methods: After confirming a lack of significant metabolic defects on 24-hour urinalysis, 23 patients with small nonobstructing unilateral nephrolithiasis detected on preoperative CT angiography underwent donor nephrectomy. Immediately after cold perfusion, ExURS was performed with ice cold saline irrigation. Retrospective review was performed.

Results: Pyeloscopy was successfully performed in all 23 patients. A total of 28 calculi, mean largest diameter $3.9 \mathrm{~mm}$ (range 3-6 $\mathrm{mm}$ ), were visualized in 19 kidneys. Basket extraction and holmium laser lithotripsy was performed in 12 and 6 kidneys, respectively. Treatment rendered 17/19 stone-containing kidneys stone free with a mean treatment time of 6.2 minutes (3-10 min). There were no intraoperative complications. Median serum creatinine level of recipients at 1 month and 1 year were $1.4 \pm 1.8 \mathrm{mg} / \mathrm{dL}$ and $1.3 \pm 0.6 \mathrm{mg} / \mathrm{dL}$, respectively. At a median follow-up of $63 \pm 47.2$ months, there were no transplant urinary calculi among the recipients.

Conclusions: ExURS safely renders live donor kidney allografts stone free with low risk of recurrence. When used appropriately, ExURS could safely increase the number of potential kidney donors and minimize the risk of adverse stone events.
\end{abstract}

\section{Introduction}

I N 2009, 16,829 KIDNEY TRANSPLANTS were performed in the United States, including 6387 from live donors. Despite this, at the time of writing this article, 85,958 patients remain on the wait list for renal transplantation (United Network for Organ Sharing/Organ Procurement and Transplantation Network data). A history of urolithiasis in the potential live donor has been considered a relative contraindication to organ donation.

In 1996, the American Society of Transplant Physicians published practice guidelines suggesting that potential donors with a history of passing a single stone with a stone-free interval of at least 10 years, low metabolic risk on 24-hour urine analysis, and negative preoperative imaging with intravenous urography may be considered for donor nephrectomy. ${ }^{1}$ The adoption of CT angiography for preoperative screening, however, has resulted in patients who are otherwise suitable donors being identified with incidental small unilateral asymptomatic nephrolithiasis. Routine CT angiog- raphy for screening of potential donors has been associated with an increase in the number of patients excluded for radiographic findings $(16 \%)$ compared with traditional modalities, including intravenous urography, renal arteriography, and renal scan $(7 \%)$, of which $42 \%$ were excluded for nephrolithiasis on CT compared with $20 \%$ with conventional imaging. ${ }^{2}$

Although these patients may be considered nonideal donors, because of the relative scarcity of available organs, many centers, including our own, have begun considering such patients for live donor nephrectomy. A recent survey of US renal transplant centers suggests that more than $75 \%$ of centers now consider some patients with a history of nephrolithiasis as live-donor candidates. ${ }^{3}$

The optimal management of stone-bearing kidneys at transplant is unknown. Some centers have opted for a conservative approach using close observation, anticipating spontaneous passage. Stone-free rates have ranged from $\sim 60 \%$ to $100 \%$ for stones $<4 \mathrm{~mm}$ in small series with $<2$ years

Department of Urology, University of Michigan, Ann Arbor, Michigan. 
mean follow-up. ${ }^{4,5}$ Conversely, Klingler and colleagues ${ }^{6}$ noted the development of ureteral obstruction after transplantation of stone-containing kidneys in $4 / 5$ patients necessitating nephrostomy tube placement, including all patients with calculi $>4 \mathrm{~mm}^{6}{ }^{6}$ Endoscopic extraction immediately after donor nephrectomy is another option that has been previously described with minimal risk of recurrence. ${ }^{7,8} \mathrm{We}$ report our experience with stone retrieval using ex-vivo ureteroscopy (ExURS) at the time of live donor nephrectomy for preoperatively identified small asymptomatic renal calculi. ${ }^{7}$

\section{Materials and Methods}

After obtaining Institutional Review Board approval, 23 potential living renal donors were identified with incidental small unilateral renal calculi on preoperative screening CT angiography between 1996 and 2010. Any history of symptomatic urolithiasis, bilateral nephrolithiasis, or incidental stone $>1 \mathrm{~cm}$ precluded patients from consideration of possible kidney donation. All donors subsequently underwent 24-hour urinalysis and were noted to be free of metabolic risk factors for urolithiasis (hypercalciuria, hypocitraturia, hyperuricosuria, hyperoxuluria). In addition, donors had negative screening results for other metabolic risk factors, including gout, hyperparathyroidism, and hypophosphatemia.

All donors were extensively counseled about the risks of nephrolithiasis developing in a solitary kidney after donor nephrectomy. Similarly, all recipients were counseled about the risk of recurrent urolithiasis developing in their denervated transplanted kidney and the need to self-monitor for signs and symptoms of recurrent urolithiasis, including hematuria and anuria.

Demographic data were obtained from donor and recipients including age, sex, location and number stones, stone size, ExURS technique, operative time, intraoperative and postoperative complications, stone-free status, stone recurrence in both donor and recipient, and renal allograft function.

\section{Donor nephrectomy and renal transplantation}

Reflecting practice pattern changes throughout the duration of the study, donor nephrectomy was performed using an open flank approach in 8 cases, an intraperitoneal hand- assisted laparoscopic approach in 12 cases, and a straight laparoscopic intraperitoneal approach in 3, of which 1 needed conversion to an open approach for adherent fat. Renal transplantation was performed by a separate transplant team in the usual manner, with the neoureterocystotomy performed using the preferred technique of the transplant surgeon. Stent placement was performed at the discretion of the transplant surgeon or if there was concern for residual stone fragments or significant edema of the ureteral stump. All patients were maintained on a cyclosporine/tacrolimus based triple drug immunosuppression regimen managed by transplant nephrology staff.

\section{Ex-vivo ureteroscopy}

Immediately after donor nephrectomy, the harvested organ was placed in ice, was flushed and perfused, and prepared for transplant per our institutional protocol. While keeping the kidney and ureter submersed in ice-cold preservation solution, ExURS was then performed by inserting the ureteroscope into the ureteral stump and advancing into the renal pelvis under direct vision. The kidney was then manipulated with the nondominant hand to align the calix of interest along the axis of the ureteroscope to minimize trauma to the procured organ (Fig. 1). Ice-cold physiologic saline irrigation was used throughout the procedure to ensure cold ischemia.

Except for the first patient, ExURS was performed without dilation of the ureteral stump. In two patients, including the first patient, a flexible $9.5 \mathrm{~F}$ ureteroscope was used. In all others, a semirigid 6.9F ureteroscope was used. In all cases, stones were identified under direct vision and managed with either basket extraction or holmium laser lithotripsy. After completion of ExURS, the kidney was provided to the transplant team for transplantation.

\section{Results}

A total of 23 patients with asymptomatic small nonobstructing nephrolithiasis identified on preoperative CT angiography underwent ipsilateral donor nephrectomy and ExURS (Table 1). The mean age of the donors and recipients was 44.0 years(range $24-67$ y) and 41.5 years (3-72),

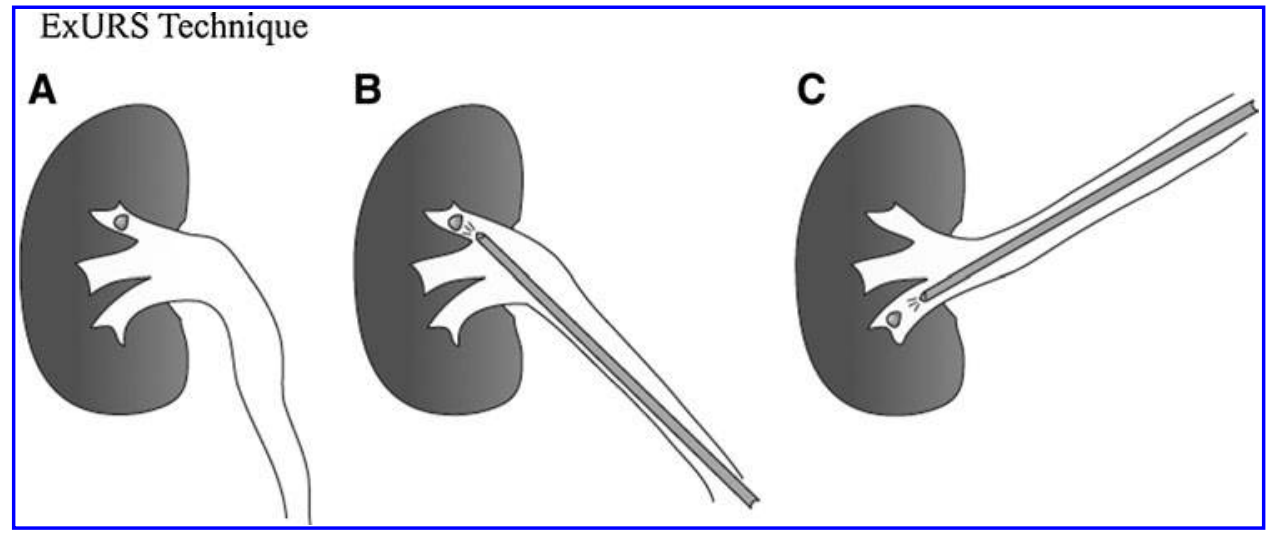

FIG. 1. (A) Harvested kidney with in situ calculus. Rotating the freed harvested kidney and ureter to align the afflicted calix in the axis of the ureteroscope allows equal, atraumatic access to both the upper pole (B) and lower pole (C) to facilitate successful endoscopic treatment. 
Table 1. Patient Demographics

\begin{tabular}{lccccc}
\hline & \multicolumn{2}{c}{ Donor } & & \multicolumn{2}{c}{ Recipient } \\
\cline { 2 - 3 } \cline { 5 - 6 } & Mean & Range & & Mean & Range \\
\hline Age (y) & 44.0 & $24-67$ & & 41.5 & $3-72$ \\
Male:female & $8: 15$ & & & $13: 10$ & \\
Largest stone size (mm) & 3.9 & $2-6$ & & \\
No. stones & 1.4 & $1-3$ & & \\
\hline Visualized stone location & \multicolumn{2}{c}{ Number } & & \\
\hline Upper pole & \multicolumn{2}{c}{5} & & \\
Midpole & 10 & & \\
Lower pole & 13 & & \\
\hline
\end{tabular}

respectively. These patients accounted for 15 living related and 8 living unrelated transplants. On preoperative imaging, a total of 32 donor stones were identified, with a mean of 1.4 (1-3) stones per donor and largest stone mean size of $3.9 \mathrm{~mm}$ $(2-6 \mathrm{~mm})$. Urolithiasis was not the etiology of end-stage renal disease in any of the recipients.

The collecting system was successfully accessed in all cases (Table 2). Dilation of the ureteral stump was performed to 20F in the initial patient, but was not determined to be necessary and was not performed in subsequent patients. A $6.9 \mathrm{~F}$ semirigid ureteroscope was used in 21 patients and a flexible ureteroscope was used in the remaining two. In four patients, no stones were visualized, consistent with either spontaneous passage of their stone before donor nephrectomy or the calcification seen on CT was intraparenchymal. In the remaining 19 patients, a total of 28 stones were visualized, of which 4 were intrapapillary and left in situ. Endoluminal basketing was used in 12 patients, and holmium:yttrium-aluminum-garnet laser lithotripsy was used in 6 patients to successfully remove/ fragment 22/24 intracollecting system stones. Overall, 17/19 stone-bearing kidneys were stone free after ExURS. The stone failures were the result of one case each of infundibular stenosis of the stone-containing calix and narrow-mouthed caliceal diverticulum. Mean operative time was 6.2 minutes (3-10 min).

No intraoperative complications were observed. Ureteral stents were placed at the time of transplant in 13/23 recipients

TABle 2. EX-VIVo Ureteroscopy Outcomes

\begin{tabular}{lcc}
\hline Outcome & Number & $\%$ \\
\hline Collecting systems accessed & $23 / 23$ & 100 \\
Kidneys with visualized stones & $19 / 23$ & 86.4 \\
Intracollecting systems stones treated & $22 / 24$ & 91.7 \\
Kidneys rendered stone free & $17 / 19$ & 89.5 \\
Ureteral stents & $13 / 23$ & 56.5 \\
& Mean & Range \\
Operative time (min) & 6.2 & $3-16$ \\
\hline Treatment technique & Number & \\
\hline Holmium laser lithotripsy & 6 & \\
Endoluminal basketing & 12 & \\
Negative ureteroscoy & 4 & \\
\hline
\end{tabular}

at the discretion of the transplant surgeon and were removed at a median of $40 \pm 17.0$ days. Postoperatively, there was one ureteral complication: Complete occlusion of the ureteroneocystotomy in a ureter without a stent. The patient underwent exploration and revision of the ureteroneocystotomy on postoperative day 4 and subsequently did well. Nonurologic Clavien class II or greater complications included wound cellulitis in one patient, lymphocele necessitating drainage in three patients, and death secondary to fatal arrhythmia/myocardial infarction just hours after transplant. ${ }^{9}$ Before death, the patient was noted to have good urine output from the transplanted kidney.

Overall, recipients had adequate graft function with a median serum creatinine level of $1.4 \pm 1.8 \mathrm{mg} / \mathrm{dL}$ on postoperative day 30 and $1.3 \pm 0.6 \mathrm{mg} / \mathrm{dL} 1$ year after transplant. One patient had delayed graft function necessitating temporary hemodialysis. One patient needed transplant nephrectomy 18 months after transplant secondary to chronic rejectionm and an additional patient needed retransplant 104 months after ExURS for chronic rejection.

Postoperative imaging studies (ultrasonography, plain radiography, and CT) were performed in all patients and reviewed to assess stone clearance and reformation. At a median follow-up of $63 \pm 47.2$ months, there have been no stone recurrences among transplant recipients. Likewise, there have been no delayed ureteral strictures among recipients.

\section{Discussion}

Routine preoperative CT angiography for screening of potential candidates for donor nephrectomy has been associated with an increase in the number of patients excluded for radiographic findings compared with traditional imaging modalities. Of these patients, greater than $40 \%$ are excluded for nephrolithiasis. ${ }^{2}$ Although previously a relative contraindication to live donor nephrectomy because of the perceived risk of the development of recurrent urolithiasis in the remaining solitary kidney of the donor as well as the denervated transplanted kidney, more than $75 \%$ of transplant centers now consider certain patients with donor lithiasis for donor nephrectomy in an effort to increase the availability of donor kidneys to meet unmet demand for renal transplantation. ${ }^{3}$ At our institution, we consider patients who are found to have small, $<1 \mathrm{~cm}$, unilateral incidental renal calculi on screening CT angiography, with no history of symptomatic urolithiasis, and normal 24-hour urine analysis for donor nephrectomy. Still, the long-term risk to graft function and optimal management of urolithiasis in renal transplantation is not well described.

The development of de novo urolithiasis in transplant kidneys is thought to occur in approximately $1 \%$ of renal transplants, with the literature reporting a range of $0.2 \%$ to $1.8 \%{ }^{10-12}$ Successful treatment of transplant lithiasis using shockwave lithotripsy (SWL) with the patient in the prone position was first reported in $1991 .{ }^{13}$ More recently, three studies have been published with 5, 8, and 13 patients demonstrating stone-free rates of $38 \%$ to $100 \%$ after a single treatment of SWL with the patient in the prone position. $6,14,15$ The pelvic location of the transplanted kidney, however, may adversely affect stone localization and targeting during SWL, hindering its effectiveness. Access to the 
transplant ureter via a retrograde ureteroscopic approach is often compromised. As a result, a number of groups have reported their experience with transplant percutaneous nephrostolithotomy for management of transplant urolithiasis. Three recent studies have demonstrated stone-free rates of $76.9 \%$ to $100 \%$ after a single procedure with no documented effects on allograft function. ${ }^{16-18}$

These studies, however, focus on management of de novo transplant lithiasis, not in situ donor gifted lithiasis. The risk of recurrent stone formation in donor gifted lithiasis is not well documented. Furthermore, the evidence guiding optimal management of donor gifted lithiasis is sparse. Some centers have reported their experience using a conservative approach of close observation. Martin and associates ${ }^{4}$ reported observing five patients with donor gifted lithiasis $<4 \mathrm{~mm}$ with spontaneous passage of $5 / 8$ stones on follow-up CT imaging at a mean of 711 days, rendering $3 / 5(60 \%)$ patients stone free without complications. Similarly, Devasia and coworkers ${ }^{5}$ reported spontaneous passage of $<4 \mathrm{~mm}$ calculi in $3 / 3$ patients on follow-up ultrasonography, with no complications and no recurrences at 1 to 2 years of follow-up. Conversely, Klingler and colleagues ${ }^{6}$ reported poor outcomes using nonsurgical expectant management. Of five patients receiving kidneys with in situ donor gifted calculi, ureteral obstruction developed in four because of stone migration, all of whom needed emergent nephrostomy tube placement. Interestingly, $2 / 3$ stones $<4 \mathrm{~mm}$ resulted in the development of ureteral obstruction.

Endoscopic extraction of in situ lithiasis in donor kidneys at the time of transplantation using a flexible cystoscope and pyelotomy has been successfully reported in three patients. ${ }^{6}$ At our institution, we have adopted the technique of ExURS immediately after donor nephrectomy, first reporting our results in 2003, which obviates the need for pyelotomy. ${ }^{7}$ Our institution continues to use this approach, having now performed ExURS in 23 patients. All such patients have been rendered functionally stone free, with the two remaining stones (one proximal to an infundibular stenosis and the other contained within a narrow mouth caloceal diverticulum) posing little to no risk of future obstruction. Importantly, all organs have been successfully transplanted with no recipient recurrences at more than 5 years of median follow-up.

To protect the procured organ, we perform ExURS after perfusion of ice-cold preservation solution, using ice-cold saline irrigation while maintaining the organ in a bath of icecold preservation solution. In our experience, ExURS adds minimal cold ischemia time, with mean time of less than 10 minutes. Furthermore, ExURS is much easier than subsequent endoscopic management because of the freedom of the nonattached ureter and kidney. Simple manipulation of the kidney and ureter to align the desired calix with the axis of the ureteroscope allows complete access to the entire collecting system (Fig. 1) with minimal risk of trauma to the transplant ureter. We have found the semirigid ureteroscope to be preferable to the flexible ureteroscope because of its shorter length, easier navigability in the freed kidney and ureter, and superior optics.

ExURS appears safe with no long-term ureteral or structural complications. A single episode of complete occlusion of the transplant ureteroneocystotomy in an anastomosis without stent in the immediate perioperative period was observed.
It is unclear if ExURS contributed to the development of this complication, or if it was related to a technical aspect of the transplant itself, or ischemia of the most vulnerable portion of the transplant ureter. Finally, renal allograft function after ExURS does not appear to be impaired, with a median serum creatinine level of $1.3 \mathrm{mg} / \mathrm{dL}$ at 1 year post-transplant in our recipients.

\section{Conclusions}

Our experience suggests that those patients with small, unilateral, incidental nephrolithiasis seen on preoperative CT angiography may safely proceed with organ donation. ExURS at the time of donor nephrectomy using either endoscopic basket extraction or holmium laser lithotripsy effectively treats in situ calculi with minimal risk to the graft and no observed de novo stone formation.

\section{Disclosure Statement}

No competing financial interests exist.

\section{References}

1. Kasiske BL, Ravenscraft M, Ramos EL, et al. The evaluation of living renal transplant donors: Clinical practice guidelines. Ad Hoc Clinical Practice Guidelines Subcommittee of the Patient Care and Education Committee of the American Society of Transplant Physicians. J Am Soc Nephrol 1996;7:2288-2313.

2. Strang AM, Lockhart ME, Amling CL, et al. Living renal donor allograft lithiasis: A review of stone related morbidity in donors and recipients. J Urol 2008;179:832836.

3. Ennis J, Kocherginsky M, Schumm LP, et al. Trends in kidney donation among kidney stone formers: A survey of US transplant centers. Am J Nephrol 2009;30:12-18.

4. Martin G, Sundaram CP, Sharfuddin A, Govani M. Asymptomatic urolithiasis in living donor transplant kidneys: Initial results. Urology 2007;70:2-6.

5. Devasia A, Chacko N, Gnanaraj L, et al. Stone-bearing live-donor kidneys for transplantation. BJU Int 2005;95: 394-397.

6. Klingler HC, Kramer G, Lodde M, Marberger M. Urolithiasis in allograft kidneys. Urology 2002;59:344-348.

7. Rashid MG, Konnak JW, Wolf JS Jr, et al. Ex vivo ureteroscopic treatment of calculi in donor kidneys at renal transplantation. J Urol 2004;171:58-60.

8. Trivedi A, Patel S, Devra A, et al. Management of calculi in a donor kidney. Transplant Proc 2007;39:761-762.

9. Clavien PA, Barkun J, de Oliveira ML, et al. The ClavienDindo classification of surgical complications: Five-year experience. Ann Surg 2009;250:187-196.

10. Shoskes DA, Hanbury D, Cranston D, Morris PJ. Urological complications in 1,000 consecutive renal transplant recipients. J Urol 1995;153:18-21.

11. Cho DK, Zackson DA, Cheigh J, et al. Urinary calculi in renal transplant recipients. Transplantation 1988;45:899902.

12. Kim H, Cheigh JS, Ham HW. Urinary stones following renal transplantation. Korean J Intern Med 2001;16:118122.

13. Wheatley M, Ohl DA, Sonda LP III, et al. Treatment of renal transplant stones by extracorporeal shock-wave lithotripsy in the prone position. Urology 1991;37:57-60. 
14. Montanari E, Zanetti G. Management of urolithiasis in renal transplantation. Arch Ital Urol Androl 2009;81:175-181.

15. Challacombe B, Dasgupta P, Tiptaft $R$, et al. Multimodal management of urolithiasis in renal transplantation. BJU Int 2005;96:385-389.

16. Rifaioglu MM, Berger AD, Pengune W, Stoller ML. Percutaneous management of stones in transplanted kidneys. Urology 2008;72:508-512.

17. Krambeck AE, Leroy AJ, Patterson DE, Gettman MT. Percutaneous nephrolithotomy success in the transplant kidney. J Urol 2008;180:2545-2549.

18. Wyatt J, Kolettis PN, Burns JR. Treatment outcomes for percutaneous nephrolithotomy in renal allografts. J Endourol 2009;23:1821-1824.
Address correspondence to: Gary J. Faerber, M.D.

Department of Urology TC 3875 University of Michigan Health System 1500 E. Medical Center Drive Ann Arbor, MI 48109-5330

E-mail: gfaerber@umich.edu

\section{Abbreviations Used \\ $\mathrm{CT}=$ computed tomography \\ ExURS $=$ ex vivo ureteroscopy \\ $\mathrm{SWL}=$ shockwave lithotripsy}



This article has been cited by:

1. François Mosimann, Mélanie Masse, Mathieu Brunet . Ex-Vivo Ureteroscopy at the Time of Live Donor Nephrectomy: A Word of Caution. Journal of Endourology, ahead of print. [Citation] [Full Text] [PDF] [PDF Plus] 